\title{
Self-perpetuating Spiral Arms in Disk Galaxies
}

\section{Citation}

D'Onghia, Elena, Mark Vogelsberger, and Lars Hernquist. 2013. “SELF-PERPETUATING

SPIRAL ARMS IN DISK GALAXIES." The Astrophysical Journal 766 (1): 34. https:// doi.org/10.1088/0004-637x/766/1/34.

\section{Permanent link}

http://nrs.harvard.edu/urn-3:HUL.InstRepos:41381806

\section{Terms of Use}

This article was downloaded from Harvard University's DASH repository, and is made available under the terms and conditions applicable to Open Access Policy Articles, as set forth at http:// nrs.harvard.edu/urn-3:HUL.InstRepos:dash.current.terms-of-use\#OAP

\section{Share Your Story}

The Harvard community has made this article openly available.

Please share how this access benefits you. Submit a story.

\section{Accessibility}




\title{
SELF-PERPETUATING SPIRAL ARMS IN DISK GALAXIES
}

\author{
Elena D’Onghia ${ }^{1,2,3}$, MARK Vogelsberger ${ }^{1}$, LARs Hernquist ${ }^{1}$
}

\begin{abstract}
The causes of spiral structure in galaxies remain uncertain. Leaving aside the grand bisymmetric spirals with their own well-known complications, here we consider the possibility that multi-armed spiral features originate from density inhomogeneities orbiting within disks. Using high-resolution N-body simulations, we follow the motions of stars under the influence of gravity, and show that mass concentrations with properties similar to those of giant molecular clouds can induce the development of spiral arms through a process termed swing amplification. However, unlike in earlier work, we demonstrate that the eventual response of the disk can be highly non-linear, significantly modifying the formation and longevity of the resulting patterns. Contrary to expectations, ragged spiral structures can thus survive at least in a statistical sense long after the original perturbing influence has been removed.
\end{abstract}

Subject headings: galaxies: kinematics and dynamics, galaxies: spiral, methods: numerical

\section{INTRODUCTION}

Seventy percent of galaxies in the nearby Universe are characterized by a disk with prominent spiral arms, but our understanding of the origin of these patterns is incomplete, even after decades of theoretical study (Toomre 1977; Athanassoula 1984, Binney \& Tremaine 2008; Sellwood 2011). Several ideas have been proposed to explain the formation of spiral arms. One model posits that these features are large-scale density waves continuing to propagate in a differentially rotating disk. In particular, this theory argues that the matter in the galaxy (stars and gas) can maintain a density wave through gravitational interactions even in the presence of shear. This density wave remains at least quasi-stationary in a frame of reference rotating around the center of the galaxy at a fixed angular speed, identified with the pattern speed of the spirals, and covers the entire disk (Lin \& Shu 1964, Bertin \& Lin 1996).

An alternative theory proposes that spiral arms are stochastically produced by local gravitational amplification in a differentially rotating disk (Goldreich \& Lynden-Bell 1965; Julian \& Toomre 1966). The mechanism behind this process is known as swing amplification and it can be seeded either by preexisting leading waves or else by the response of a disk to the presence of a corotating overdensity, such as a giant molecular cloud. This dynamical response takes the form of wakelets in the surrounding medium, each amplified by its own self-gravity through the swinging of leading features into trailing ones owing to the shear.

According to this second theory, spiral arms would fade away in one or two galactic years if the driving perturbations were removed (Toomre \& Kalnajs 1991), in contrast to the quasi-steady nature of the arms in the model proposed by Lin and Shu. Thus, a continuous source of perturbations would be required for these fluctuating spiral patterns to be maintained throughout the lifetime of a galaxy. Indeed, by mimicking the effects of dissipative infall of gas, Sellwood \& Carlberg (1984) showed that the addition of fresh particles on circular orbits could cause such spiral patterns to recur, and Carlberg

\footnotetext{
${ }^{1}$ Harvard-Smithsonian Center for Astrophysics, 60 Garden Str., Cambridge, MA 02138 USA

2 Keck Fellow; edonghia@cfa.harvard.edu

${ }^{3}$ University of Wisconsin,

475 Charter St., Madison, WI 53706
}

\& Freedman (1985) demonstrated that almost any mechanism of dynamical cooling can maintain spiral activity of a similar kind.

However, the pioneering work on density waves in the 1960s was based on analytic theory and invoked various assumptions in order for solutions to be found. For example, the swing amplification analysis involved linear approximations to the equations of motion. The theoretical emphasis since that time has been on identifying driving mechanisms that can sustain the wave in spite of the damping which would cause it to decay in this picture. Indeed, whereas observations show that spiral arms might be density waves, N-body experiments have not yielded long-lived spiral structures, as predicted by the stationary density wave theory. Simulations of cool, shearing disks always exhibit recurrent transient spiral activity and this situation has not changed over the past several decades as computational power has increased (Sellwood 2000, Fujii et al. 2011; Sellwood 2011).

Some work showed that spiral patterns fade away in numerical simulations of stellar disks if the effects of gas dissipation are not included; the reason is that the disk becomes less responsive as random motions rise owing to particle scattering by the spiral activity and giant molecular clouds (Sellwood \& Carlberg 1984; Binney \& Lacey 1988). Moreover, the debate about the longevity of the arms practically ceased two decades ago because the available computational power did not permit definitive tests of some of the predictions of the theories and also because observations at that time were not sufficiently detailed to discriminate between the two main competing views.

In the past decade, some studies have argued that the continuous infall of substructures in the dark matter halos of galaxies could induce spiral patterns in disks by generating localized disturbances that grow by swing amplification (Gauthier et al. 2006; Purcell et al. 2011). According to such simulations, the main agent producing transient features would be satellite passages through the inner part of a disk. Because the tidal effects of the satellites are generally small (D'Onghia et al. 2010b), this process is distinct from interactions thought to be responsible for grand-design spirals like M51. However, there are indications that dark matter substructures orbiting in the inner regions of galaxy halos would be destroyed by dynamical processes such as disk shocking, and hence would not be able to seed the formation of spiral structure (D'Onghia et al.2010a). 
In what follows, we report on high resolution N-body simulations of isolated disks in which we follow the motion of 100 million star particles under the influence of gravity, to explore the idea that spiral arms are seeded by density inhomogeneities orbiting within the disk. These inhomogeneities can be identified with fluctuations in the distribution of gas in the interstellar medium of galaxies, such as giant molecular clouds or, more speculatively, any massive star clusters embedded in the disk. Because early works already showed that the gravitational response of a stellar disk to an overdensity orbiting in its plane is similar as the response of a gaseous disk (see in particular Fig.7 of Toomre (1981)), we perform our analysis only on stellar disks, for simplicity.

Our work focuses on understanding the origin of spiral arms and differs in many respects from previous studies. The methodology we employ here has the potential to discern the physical processes occurring in stellar disks at a higher level of detail than in previous simulations of isolated galaxies.

As we describe below, our results indicate that the response of a disk to local perturbations is highly non-linear and timevariable on galactic scales and so is not fully captured by the linear approximations invoked in swing amplification theory or the classic work on quasi-steady density waves.

In what follows, we describe our methodology in $\S 2$. In $\S 3$ we present our results by showing examples in which a disk is perturbed by inhomogeneities orbiting in its plane, and we even vary the lifetimes of the perturbers. Results anticipated by linear theory are described in $\S 4$, whereas $\S 5$ is devoted to illustrating the non-linear effects that arise in our simulations. Finally, we discuss the astrophysical relevance of our work in $\S 6$.

\section{METHODOLOGY}

Our simulations were carried out with the parallel TreePM code GADGET-3 (last described in Springel 2005). We only employ the tree-based gravity solver coupled to a static external potential to solve for the evolution of collisionless particles.

Pairwise particle interactions are softened with a spline kernel (Hernquist \& Katz 1989) on scales $h_{s}$, so that forces are strictly Newtonian for particles separated by more than $2 h_{s}$. The resulting force is roughly equivalent to traditional Plummer-softening with scale length $\epsilon \approx h_{s} / 2.8$. For our simulations the Plummer-equivalent gravitational softening length set to $\epsilon=5 \mathrm{pc}$ for stellar particles.

\subsection{Setting up initial conditions}

The galaxies in our study consist of dark matter halos and rotationally supported disks of stars. The parameters describing each component are independent and the models are constructed in a manner similar to the approach described in previous works (Hernquist 1993, Springel 2000; Springel et al. 2005).

\subsection{Dark Halo}

We model the dark matter mass distribution with a Hernquist (1990) profile:

$$
\rho_{\mathrm{dm}}=\frac{M_{\mathrm{dm}}}{2 \pi} \frac{a}{r(r+a)^{3}},
$$

which has a cumulative mass distribution $M(<r)=M_{\mathrm{dm}} r^{2} /(r+a)^{2}$, where $a$ is the radial scale length and $M_{\mathrm{dm}}$ is the total halo mass here set to $9.5 \times 10^{11}$ $\mathbf{M}_{\odot}$.
In the past, models of disk galaxies run in isolation and used to study the properties of spiral arms employed only a few million particles to sample both the stellar disk and the dark matter halo. In such experiments, randomly-placed particles produce fluctuations in the halo potential. Even if the disk is initially featureless, the Poisson noise owing to such discretization of the mass in N-body experiments is inevitably swing amplified, producing trailing multi-armed spiral patterns in the disk (Toomre 1977; Fujii et al. 2011; Sellwood 2012).

In order to suppress the development of artificial features in all the $\mathrm{N}$-body experiments that follow, we set up a live disk of stars embedded in a rigid dark matter potential. We employ simulations with a sufficiently large number of particles in the disk, i.e. 100 million, so that the disks are essentially featureless when evolved without any perturbers acting on them. These simulations serve as "controls," making it possible to identify the response of the disk to imposed perturbations. In this manner, we will be able to separate the sources responsible for exciting features in the disk from the stars which react to the perturbations, unlike previous experiments in which the stars themselves acted as perturbers, complicating the interpretation of the experiments, as emphasized by Toomre (1990).

\subsection{Stellar Disk}

We model the stellar disk in the initial conditions with an exponential surface density profile of scale length $R_{s}$ :

$$
\Sigma_{*}(R)=\frac{M_{*}}{2 \pi R_{s}^{2}} \exp \left(-\mathrm{R} / \mathrm{R}_{\mathrm{s}}\right),
$$

so that the stellar disk mass is $M_{*}=m_{d} M_{\text {tot }}$, where $m_{d}$ is the disk fraction of the total mass $M_{\text {tot }}$ of the galaxy. For an extremely thin disk the circular velocity of the galaxy would be:

$$
\begin{array}{r}
V_{c}^{2}(R)=\frac{G M_{\mathrm{dm}}(<R)}{R}+\frac{2 G M_{*}}{R_{s}} y^{2} \times \\
{\left[I_{0}(y) K_{0}(y)-I_{1}(y) K_{1}(y)\right] .}
\end{array}
$$

Here, $G$ is the gravitational constant, $y=R /\left(2 R_{s}\right)$, and $I_{n}$ and $K_{n}$ are modified Bessel functions. We specify the vertical mass distribution of the stars in the disk by giving it the profile of an isothermal sheet with a radially constant scale height $z_{0}$. The 3D stellar density in the disk is hence given by:

$$
\rho_{*}(R, z)=\frac{M_{*}}{4 \pi z_{0} R_{s}^{2}} \operatorname{sech}^{2}\left(\frac{\mathrm{z}}{\mathrm{z}_{0}}\right) \exp \left(-\frac{\mathrm{R}}{\mathrm{R}_{\mathrm{s}}}\right) .
$$

We treat $z_{0}$ as a free parameter that is set by the vertical velocity dispersion of the stars in the disk and fix the velocity distribution of the stars such that this scale height is selfconsistently maintained in the full 3D potential of the galaxy (Springel et al.2005). We adopt $z_{0}=0.1 R_{s}$.

Fig. 11 shows the rotation curve for the fiducial galaxy model used in the simulations (left panel). The total disk mass of the galaxy is $1.9 \times 10^{10} \mathrm{M}_{\odot}$. The total disk fraction is fixed to $m_{d}=0.02$ and the scale length of the disk, $R_{s}$, is 3.13 $\mathrm{kpc}$. The disk is set up so that it is stable, as measured by the $Q$ parameter, defined for infinitely thin disks as:

$$
Q=\frac{\sigma_{R} \kappa}{3.36 G \Sigma}
$$

where $\sigma_{R}$ is the radial velocity dispersion, $\kappa$ is the epicycle frequency, and $\Sigma$ is the stellar surface density. The properties of the disk are chosen so that the $Q$ parameter is initially 

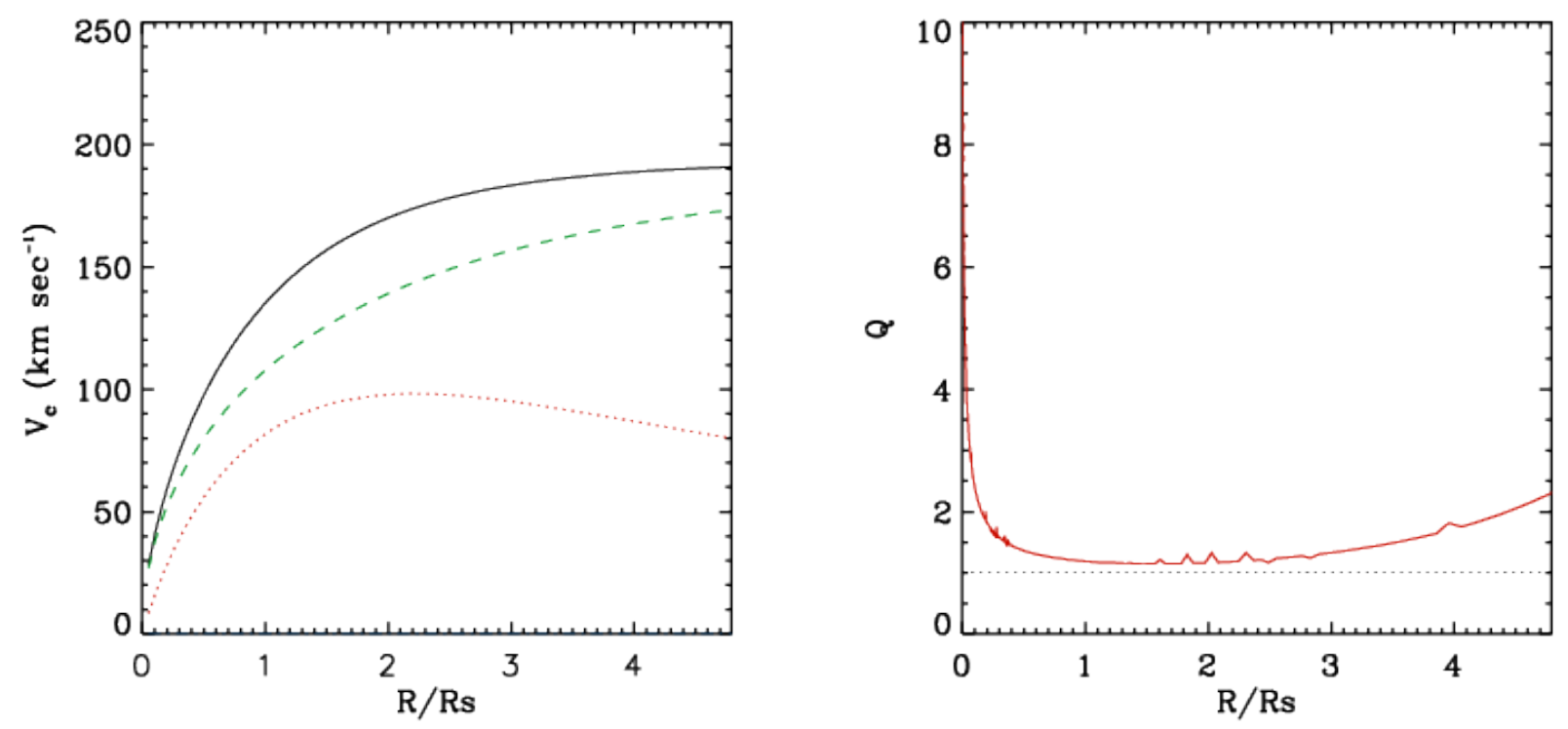

FIG. 1.- Left Panel.Rotation curve for fiducial disk galaxy model (left panel). Right Panel. Toomre parameter $Q$ as a function of radius for the fiducial model. The radius is normalized to the value of the disk scale length of $3.13 \mathrm{kpc}$ in both panels.

larger than 1 at all radii and has a minimum value of 1.3 (right panel of Fig 1), implying that the disk is stable to axisymmetric instabilities (Toomre 1964).

The strength of spiral features is estimated by dividing the face-on disk into concentric annuli which are further divided into azimuthal bins. The mass from the star particles is then assigned to these bins, making it possible to compute the stellar surface density $\Sigma(R, \theta)$. Fourier components of the surface density are then calculated. We use the Fourier transform analysis to estimate the residuals of the surface density computed by subtracting the azimuthally averaged surface density from the surface density distribution and normalizing according to Res $=\left(\Sigma-\Sigma_{\mathrm{avg}}\right) / \Sigma_{\mathrm{avg}}$.

\section{RESULTS}

\subsection{Disk Stability Test}

We first perform an N-body experiment in which a live disk of 100 million stars as described above is embedded in a rigid potential for the dark halo. We run the fiducial case with no perturbers for two-three galactic years and verify that the disk does not develop prominent spiral features. The outcome of this experiment is shown in Fig. 2 where a time evolution of the disk is displayed.

\subsection{Dynamical Response of the Disk to Perturbers}

Having established that relatively low mass disks run with sufficiently large numbers of particles do not develop prominent spiral patterns in the absence of any perturbing influence, we then evolve the same galaxy models, but add 1,000 gravitationally softened particles (each with a mass of the order of a typical giant molecular cloud: $\mathrm{M}=9.5 \times 10^{5} \mathrm{M}_{\odot}$ ) distributed with the same exponential law as the disk and assumed to be corotating on circular orbits with the disk stars. We note that we use a 20 times larger gravitational softening length for these molecular cloud particles due to their larger mass compared to the stellar particles in the disk. These perturbers are kept moving on circular orbits in order to avoid any clumping that could facilitate the formation of overdense stellar regions.

Each softened particle perturbs the motions of neighboring stars and drives a strong local response in the disk. The col- lective influence of all the perturbers leads to the development of spiral structures as shown in Fig. 3. These features extend well beyond two scale lengths of the disk. At a distance of five scale lengths these segments tend to be more isolated and weaker.

In particular, Fig. 3 displays the time evolution of the disk for approximately two-three galactic years. Note that the dynamical response is rapid and already after almost half of a galactic year, multi-armed patterns appear. Fig. 4(top panels) shows the disk and the 1,000 molecular clouds distributed within it after approximately one galactic year. The disk is displayed face-on in Cartesian coordinates (left panels) and surface density residuals are shown in polar coordinates (right panels). In this case, interior to one disk scale length the disk is characterized by three or four spiral arms. At two scale lengths from the galaxy center the number of spiral arms is approximately seven with amplitudes reaching values of 10$15 \%$ higher than the stellar background.

We repeat the same experiment with the giant molecular clouds counter-rotating relative to the disk stars. As shown in Fig. 4 (bottom panels), the disk response after approximately one galactic year is now much weaker than for corotating perturbers displayed at the same time (top panels).

\subsection{Finite Lifetime Giant Molecular Clouds}

Our previous calculations assumed an infinite lifetime for the giant molecular clouds. Although the lifetimes of these systems are much debated, giant molecular clouds in galaxies are likely short-lived. Furthermore, giant molecular clouds in real galaxies are preferentially found in spiral arms, and may be created there by the converging flow of the interstellar medium due to its motion through the spiral potential. The clouds disperse after they pass through the arm, perhaps in part because of the diverging flow as the gas emerges from the arm, although other factors such as of the energy input from young stars are important also. This is certainly a too complicated picture to be simplified, however here we only want to measure the dynamical response of the disk to some perturbers with different lifetimes.

In the following, we show the outcome of runs performed with a finite lifetime for the giant molecular clouds. In partic- 

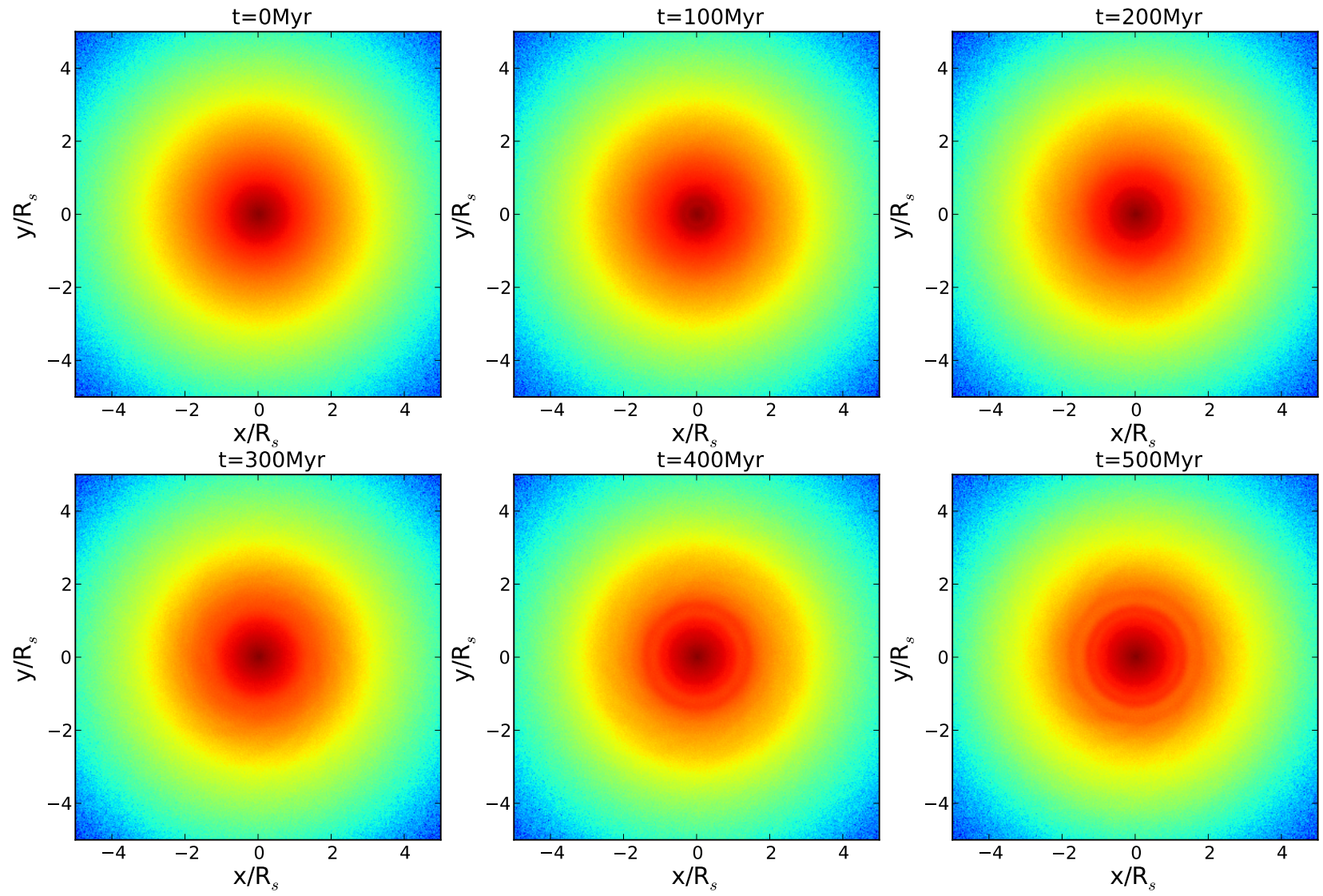

FIG. 2.- Time sequence for the evolution of a live disk of stars embedded in a dark Milky Way-sized halo run unperturbed in isolation and displayed after a few galactic years. The disk is displayed face-on in Cartesian coordinates.

ular, we show two examples: a first run adopting the fiducial disk of stars embedded in a rigid halo potential with 1,000 giant molecular clouds having a lifetime of $2.5 \times 10^{6} \mathrm{yrs}$ and a second run with cloud lifetimes of $2.5 \times 10^{7} \mathrm{yrs}$. The finite lifetime is directly implemented in our simulation code. Specifically, we assign a time counter to each giant molecular cloud particle. Once this counter exceeds the predefined lifetime, this particle is converted to a regular stellar particle by changing its mass and gravitational softening length. We then randomly select another stellar particle in the disk and turn it into a giant molecular cloud particle also by changing the mass and gravitational softening length accordingly. To avoid the sudden destruction of all giant molecular cloud particles at the same time, we generate the initial set of giant molecular cloud particles with a uniform age distribution in the range of the selected lifetime.

The top panels of Fig. 5 show the outcome of the run with the giant molecular clouds having a lifetime of $2.5 \times 10^{6} \mathrm{yrs}$. This lifetime is too short to trigger any resonance between the shear flow and epicyclic vibration, hence the forced response that leads to the formation of the arms does not occur. The opposite situation is shown in Fig. 5(bottom panels) where the giant molecular clouds are assumed to live for $2.5 \times 10^{7} \mathrm{yrs}$. Note that this lifetime is sufficient to trigger the formation of multi-armed spiral patterns. The orbital period for a typical giant molecular cloud in a Milky-Way sized galaxy is $\approx 10^{8}$ yrs, thus our calculations show that a lifetime of a quarter of the orbital time is sufficient to trigger a strong response in a disk and the subsequent development of spiral arms. The argument can be now inverted: in order to form realistic spiral arms in disk galaxies, the giant molecular clouds have to sur- vive for at least $10^{7} \mathrm{yrs}$, which is in accord with observational estimates.

\section{RESULTS ANTICIPATED BY LINEAR THEORY}

\subsection{Material Arms or Density Waves?}

A long-standing controversy over the nature of spiral arms is whether they correspond to density enhancements in the background stellar distribution (density waves), or are made up of stars that always remain in the arm and are just more concentrated than the stars outside the arm (material arms). In the early studies, the arms were assumed to be density waves, because if they were material they would quickly wind up as the galaxy rotates. Thus, both the swing amplification and the static density wave theories argue that the arms are overdense regions of the disk moving around at a different speed relative to the stars themselves. Stars thus continuously move in and out of the spiral arms. However, recent investigations using numerical simulations of stellar disks have challenged this claim and and argue that the arms might be material structures (Grand et al.2012).

To investigate this in the context of our simulations, we identify a patch of stars along the arm in the stellar disk after the arms are fully developed (after 100 Myrs) as displayed in top panels of Fig. 6. where the patch is colored in black. Then, we follow the positions of the stars originally in the patch forward in time and display the outcome after two galactic years (bottom panels). We note that the stars initially in the patch spread out, confirming that the spiral patterns in our simulations are density waves and not material structures. This is shown in polar coordinates in the bottom panel of Fig. 6 where it is clear that the patch is being sheared out by differ- 

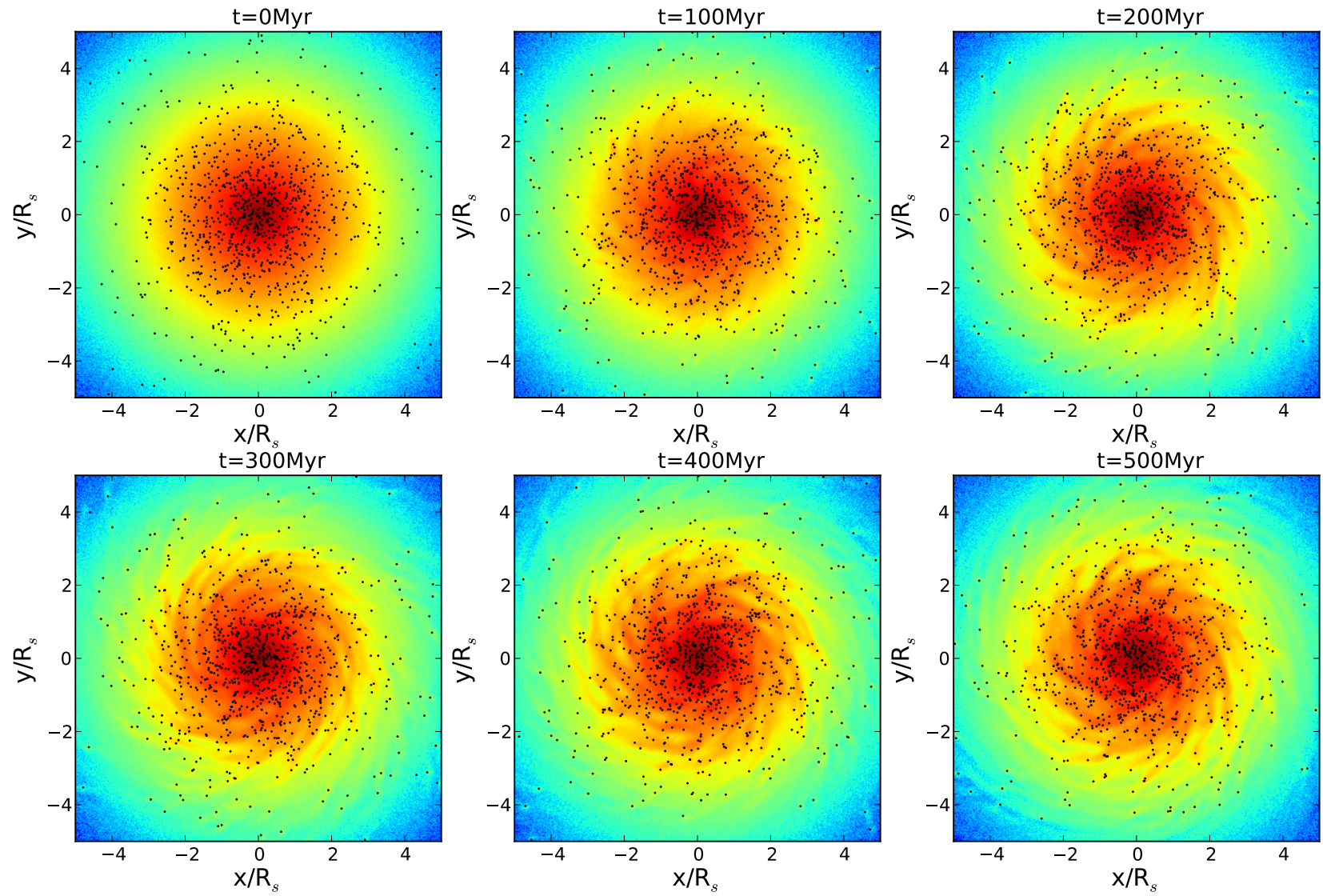

FIG. 3.- Time sequence of an N-body experiment in which a self-gravitating disk of 100 million stars seen face-on embedded in a dark Milky Way-sized halo is run with 1,000 giant molecular clouds (represented with the black dots) which are randomly distributed within the disk and are assumed to be corotating on circular orbits with the disk stars. The giant molecular clouds act as perturbers and the live disk dynamically responds to the presence of these perturbers by developing features which resemble multi-armed structures in galaxies. Each panel displays a region $30 \mathrm{kpc}$ on a side at the times indicated.

ential rotation and the pitch angle of the patch differs significantly from that of the spiral features.

\subsection{Forced-Wake Theory}

The different response of the disk to the direction of the motion of the perturbers within the disk, as shown in Fig. 4 suggests that the physical process behind spiral arm formation in our simulated galaxies is initially, at least, wake-making, which operates through a combination of three ingredients: the shearing flow, small-scale epicyclic motions, and the disk self-gravity (Julian \& Toomre 1966). We briefly review the main concept.

Consider stars drifting in the flow at slightly larger radii than a perturber which is corotating with the disk. The drifting stars will feel a small force owing to the presence of the perturber, exciting epicyclic motion in the stellar orbits. At the same time, the inwards and outwards motions of the stars lead to density enhancements and deficits relative to the stellar background. These enhancements can swing from leading to trailing owing to shear in the disk. The sense of this shearing motion is identical to that of the retrograde epicyclic motion of individual stars. Because of this match it is possible for a swinging pattern to resonate with the epicyclic stellar motions as the shear causes it to become a trailing pattern. This temporary match allows the feature to be amplified by self-gravity as the stars linger within the perturbation (Julian \& Toomre 1966).

\subsection{The Critical Role of Self-gravity as Amplifier}

Julian \& Toomre (1966) showed that swing amplification results from a cooperative effect between shear, epicyclic motions, and self-gravity. In order to verify the importance of self-gravity in the outcomes of our simulations, we performed an N-body simulation of a disk of stars treated as test particles embedded in a dark halo along with 1,000 perturbers moving on circular orbits within the disk (for details of the set-up, see Appendix).

Fig. 7 shows a time sequence from a simulation in which the disk is perturbed by density inhomogeneities, but the selfgravity of the disk is ignored. The influence of the disk selfgravity is clear. In the absence of self-gravity, wakes still form around the perturbers, but the wakes have different shapes and are weaker than when self-gravity is included, as demonstrated in Fig. 7. A harmonic analysis shows that the magnitude of each Fourier coefficient is greatly reduced if disk selfgravity is ignored, as expected on the basis of similar tests performed by Julian \& Toomre (1966). (In particular, compare Figs. 7 and 12 in Julian \& Toomre (1966).) Thus, selfgravity acts as a communicator between the stars, amplifying wakes around each perturber and allowing different wakes to connect to produce long-range patterns, as in Fig. 3 .

\subsection{Collective Amplification Regime}

Swing amplification is the linear gravitational response of a disk to an overdensity orbiting in its plane. In a stable disk, pressure-like or mixing effects of random motions avoid clumping and normally disrupt an overdense region before it has time to collapse. Because of the similarity between 

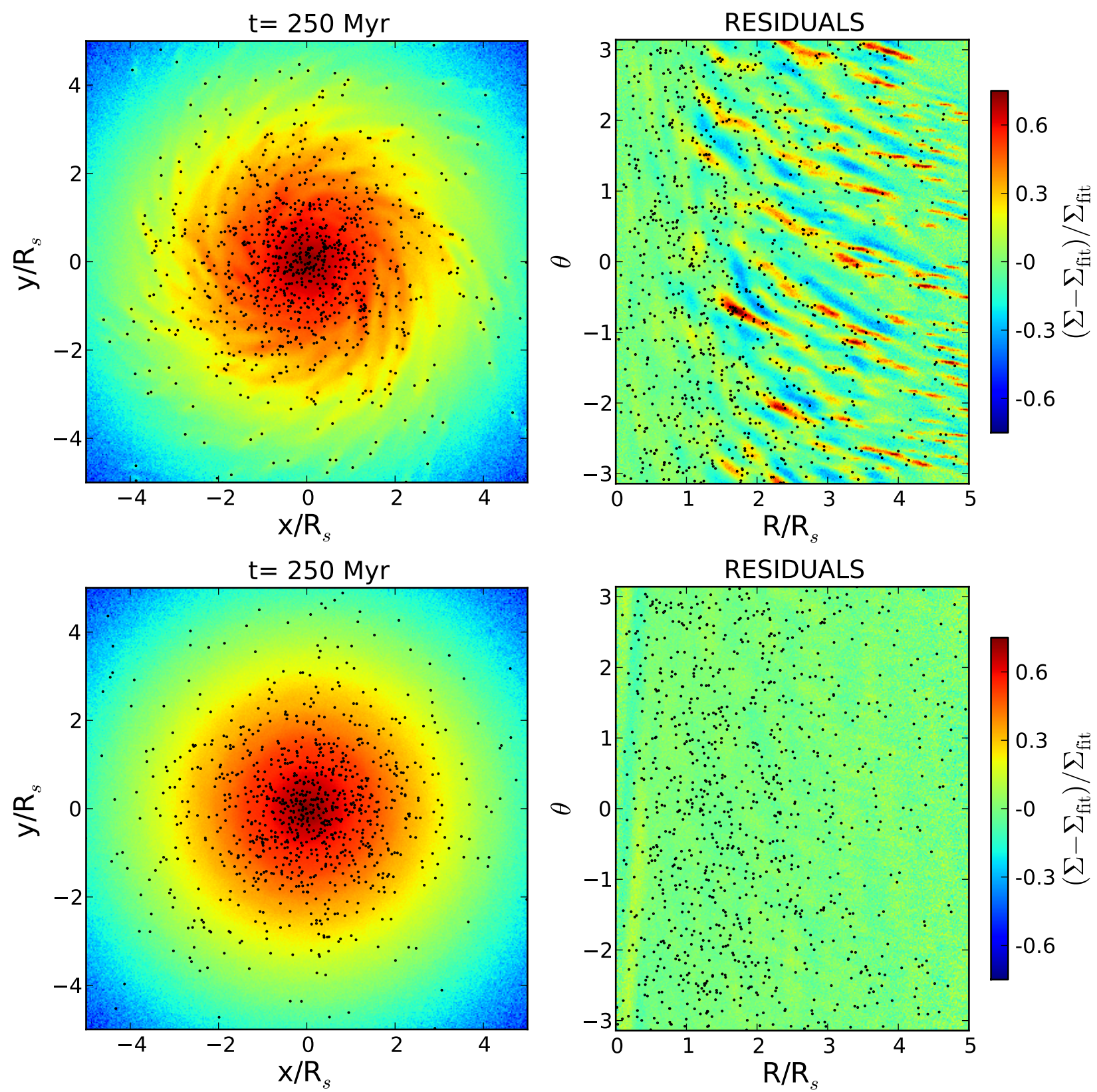

FIG. 4.- Top Panels.A live disk of stars embedded in a dark Milky Way-sized halo run in isolation with 1,000 giant molecular clouds distributed within the disk and assumed to be corotating on circular orbits with the disk. The disk is displayed approximately after one galactic year face-on in Cartesian coordinates (left) and surface density residuals are shown in polar coordinates (right). Bottom Panels. The same experiment is displayed at the same time with the 1,000 perturbers assumed to be counter-rotating on circular orbits with respect to the disk.

shear and epicyclic vibrations however, individual stars remain within shearing density enhancements for a substantial fraction of the epicyclic frequency $\kappa$, which is given by

$$
\kappa^{2}=R \frac{\mathrm{d} \Omega^{2}}{\mathrm{dR}}+4 \Omega^{2}
$$

where $\Omega=V_{c} / R$ is the angular frequency, $R$ is the radius, and $V_{c}$ is the total circular velocity. For the idealized case of a Mestel disk, with $V_{c}$ constant, Toomre (1981) found that the gain of the swing amplifier depends on the value of $Q$ and the ratio of the wavelength to a critical value $X=\lambda / \lambda_{\text {crit }}$ where $\lambda_{\text {crit }}=4 \pi G \Sigma \kappa^{-2}$ is the shortest wavelength stabilized by rotation alone. For this idealized situation, the amplification factor peaks at $X \sim 1.5$, reaching values in excess of 100 for cold disks. Swing amplification is negligible for $X \geq 3$.
Indeed perturbations with such long wavelengths are stabilized by the angular momentum of the stars. Note that in N-body experiments, particle noise creates a spectrum of perturbations of all sizes and shapes, including both leading and trailing spirals. As the differential rotation of the disk shears leading spirals into trailing configurations, swing amplification boosts the amplitudes of those which have wavelengths of order of $\lambda \sim 1.5 \lambda_{\text {crit }}$ thereby creating a multi-armed pattern of trailing spirals with a characteristic spacing that explains the results illustrated in Fig. 3 (see Appendix for an expression giving the amplification parameter for a general disk galaxy).

A naive expectation from Julian \& Toomre (1966) is that each inhomogeneity in the disk should generate a wake, forming an initial leading feature which is amplified owing to the 

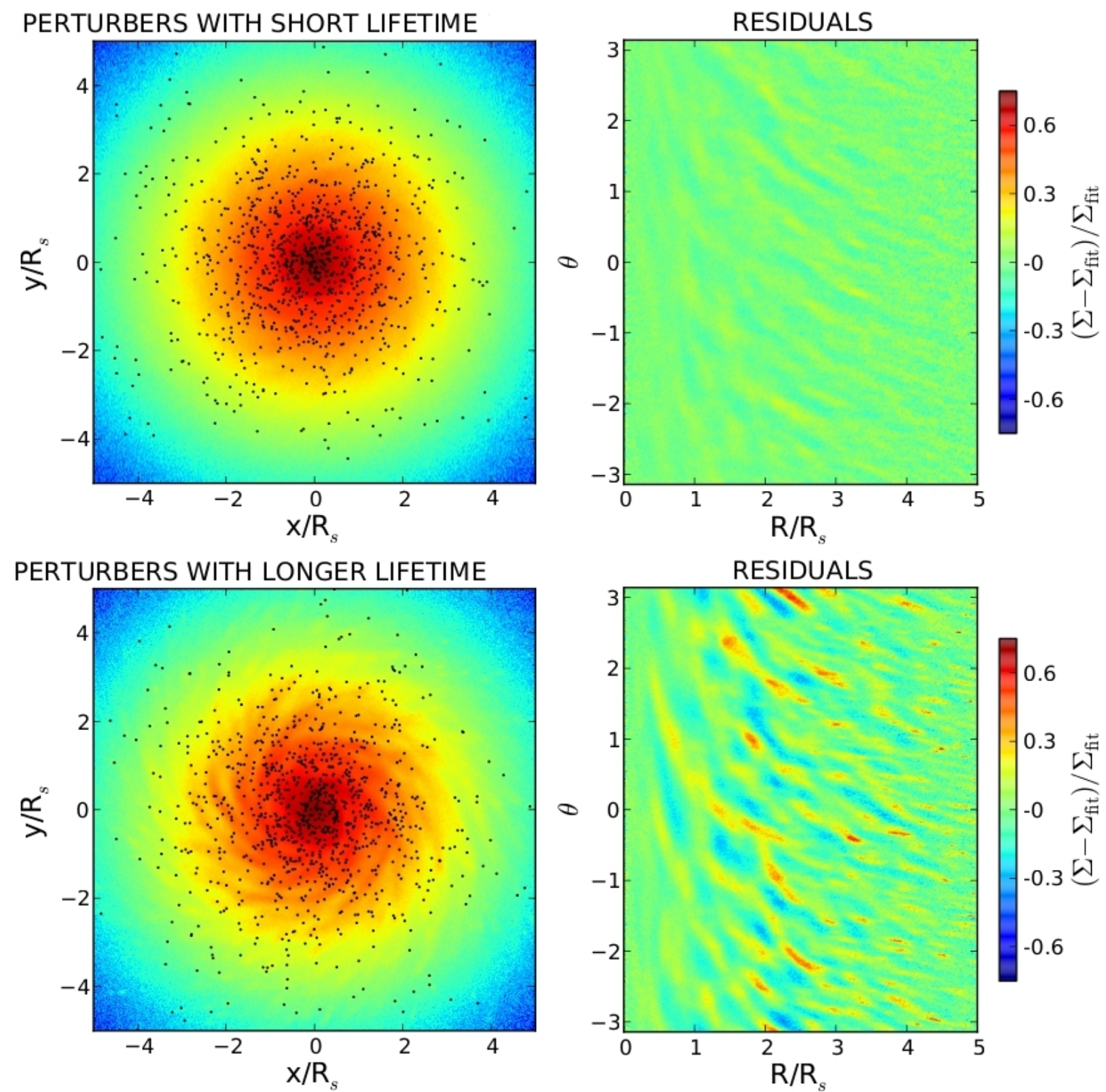

FIG. 5.- Left Panels. The outcome of the N-body experiment where a live disk of stars embedded in a dark Milky Way-sized halo run with 1,000 giant molecular clouds with a finite lifetime of $2.5 \times 10^{6}$ yrs (top) and $2.5 \times 10^{7}$ yrs (bottom). Right Panels. The surface density residuals are shown in polar coordinates.

differential rotation of the disk to become a stronger trailing wave. However, in our simulations the number of arms is not determined by the number of perturbers. ${ }^{4}$ Instead, each perturber produces a segment of an arm and then these segments are joined at kinks. Thus, there is a collective process that occurs between the individual wakes that connects them together to produce a pattern that resembles the features in multi-armed spiral galaxies.

According to swing amplification theory, the most strongly amplified features are those which have wavelength $\sim \lambda_{\text {crit }}$. If in some region the perturbers are packed together more tightly than this, they will effectively act as an individual perturber producing a single response with an extent $\sim \lambda_{\text {crit }}$. In regions where the perturbers are separated by distances much

\footnotetext{
${ }^{4}$ It should be noticed that in linear theory, there is fundamentally no difference, whether or not the perturbers are distinct from the supporting medium (Toomre 1990).
}

larger than $\lambda_{\text {crit }}$, they are effectively isolated, and we would expect to see individual, disconnected wakes around each perturber. However, in the intermediate regime, when the density of the perturbers is such that they can each produce wakes that interact with one another, the wakes will connect owing to self-gravity, joining together to produce patterns that can cover an entire disk. This has the effect of selecting out particular global structures that extend throughout a disk and the resulting number of arms is governed by the structural properties of a galaxy through $\lambda_{\text {crit }}$.

\subsection{Determination of the Number of Arms}

So far we have presented models of disks of such mass that when they are perturbed they develop features characteristic of multi-armed spiral galaxies. We have verified that the number of arms does not depend on the number of perturbers. However, the response does depend in detail on the mass distribution of the galaxy and the extent to which the 

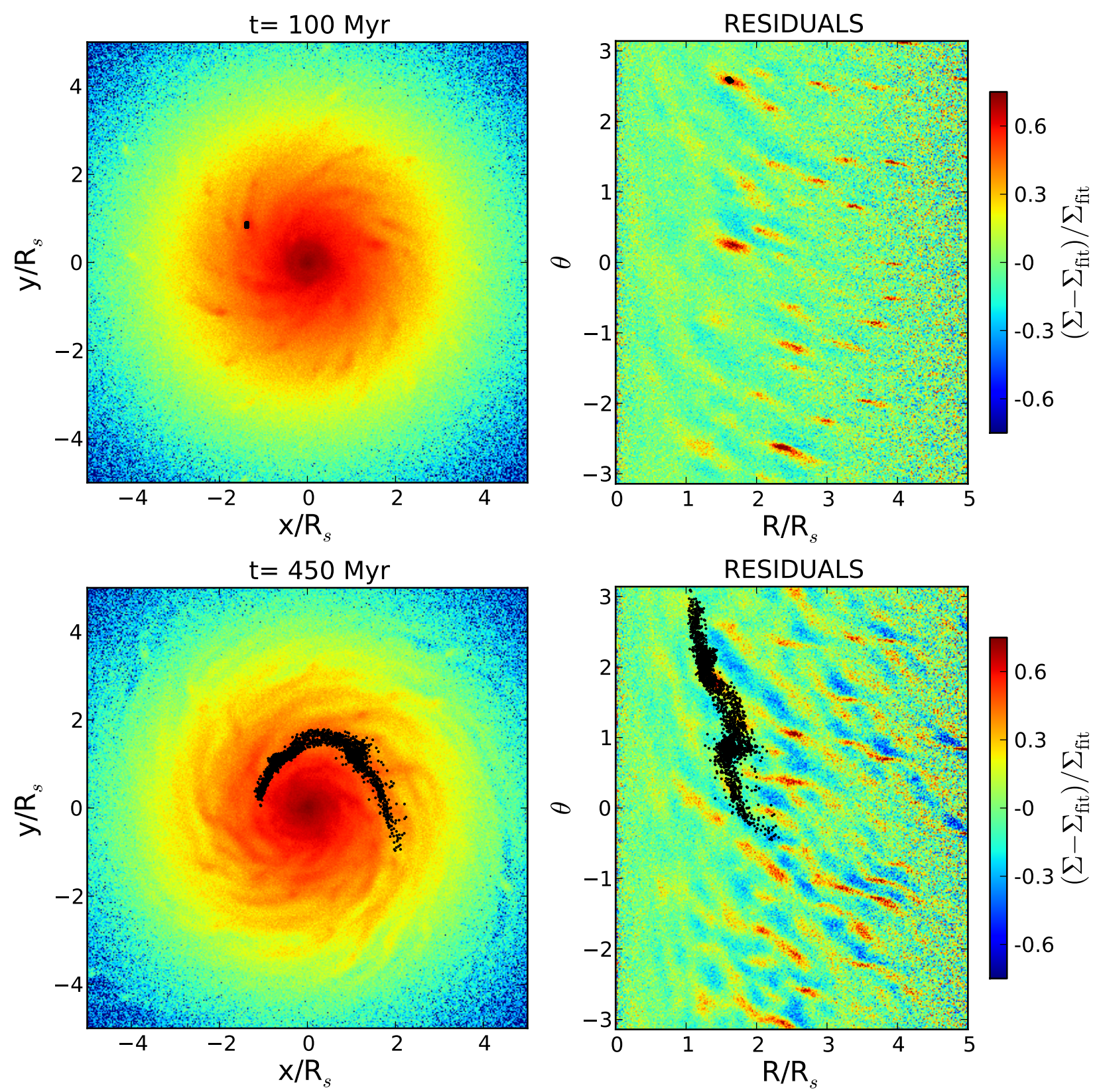

FIG. 6.- Top Panels. A patch of stars is identified in the overdense region of the arm and colored in black at the time when the arms are fully developed (100 Myrs). Bottom Panels. The stars initially identified in the patch are followed forward in time and displayed after less than two galactic years.

disk is self-gravitating.

In a set of experiments shown in Fig. 8, we altered the contribution of the disk to the total mass of the galaxy. We kept the total mass of the galaxy and the mass distribution of the dark halo as previously but we ran simulations where we increased the disk fraction, $m_{d}$, from 0.01 to $0.02,0.03$ and 0.04. Once again, we first ran these models without perturbers orbiting within the disk for one galactic year and verified that the disks do not develop prominent patterns. We subsequently introduced in the initial conditions 1,000 giant molecular clouds corotating with the disk stars. By changing the disk mass these models were designed to vary the critical length scale parameter $\lambda_{c r i t}=4 \pi^{2} G \Sigma / \kappa^{2}$ and lead to a different spiral morphology (see Fig. 1 in Carlberg \& Freedman (1985); Efstathiou et al. (1982)), from the multi-armed features typical of galaxies like NGC 7217 obtained for low- mass disks, to those with a few prominent arms as in M101 or the Milky Way. As anticipated based on the above arguments, the results shown in Fig. 8 demonstrate that the spiral morphology in our simulated disks is indeed determined by the structural properties of the galaxy through $\lambda_{\text {crit }}$.

\section{RESULTS NOT ANTICIPATED BY LINEAR THEORY}

\subsection{Self-perpetuating spiral arms}

Theories based on a linear approximation describing the local dynamic response, e.g. a disk patch, predict that the emerging spiral patterns should be a superposition of swinging wavelets, shearing with the general flow, but exhibiting transient growth as they swing from leading to trailing. If the perturbation responsible for exciting a wakelet is removed, the linear theory predicts that the wakelet will decay, revealing its transient nature (Toomre \& Kalnajs 1991). In order to 

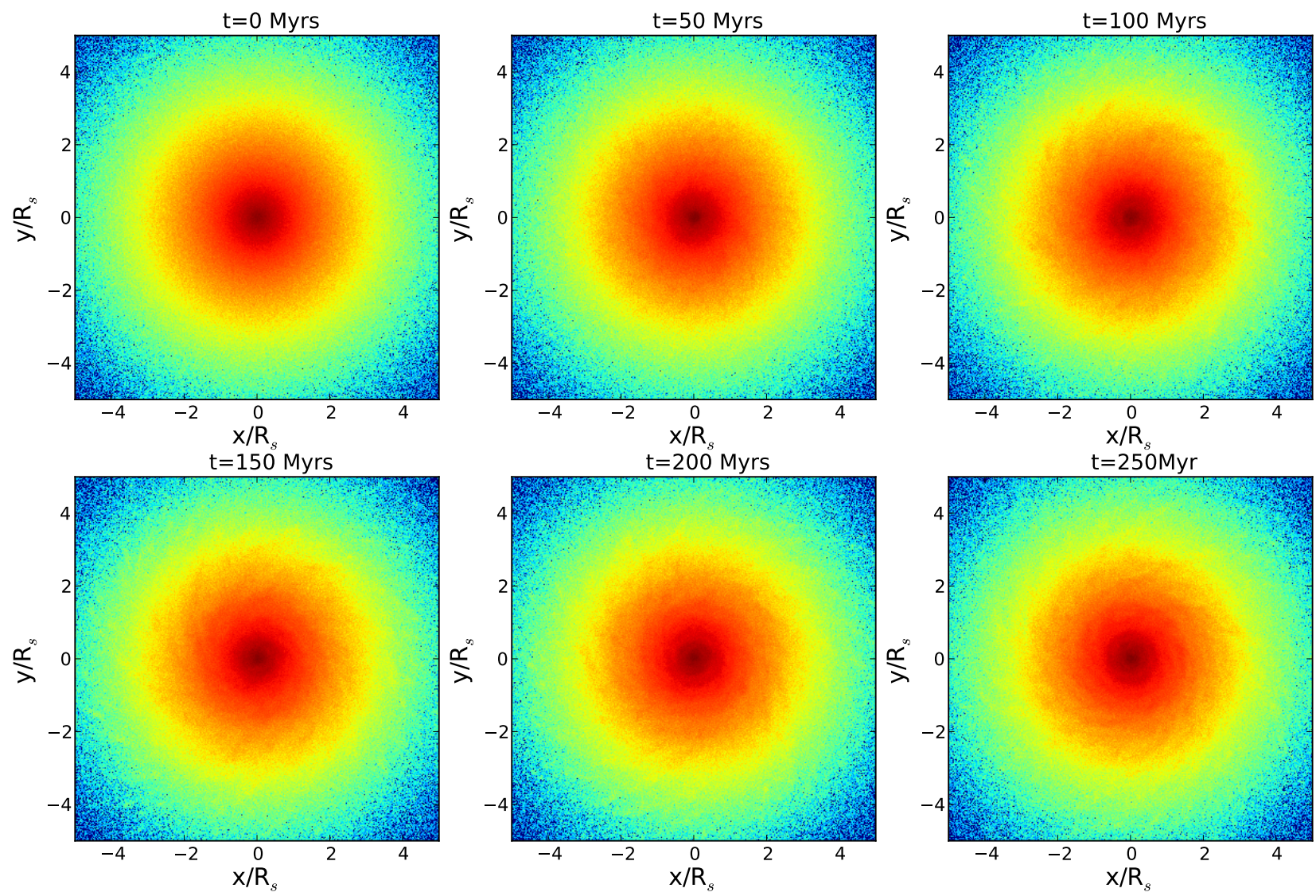

FIG. 7.- Time evolution of a disk perturbed by 1,000 inhomogeneities co-rotating with the stars, but in which the disk self-gravity is not included.

test the validity of the linear theory and to determine if spiral structures arise collectively through a local response of the disk, we stacked the giant molecular clouds at a fixed radius in the disk, superposing their associated wakes. The outcome is surprising, and we find that the response is highly non-linear in the sense that the wakes that initially formed around the perturbers, e.g. the giant molecular clouds, depart from them after only about a tenth of a galactic year and then become new perturbers that continuously excite the disk.

In fact, this is apparent in the top right panel of Fig. 4 where it can be seen that at a fixed radius between say 2 and 4 disk scale-length, the wakelets have different shapes and cannot be superposed. Furthermore, the perturbers (the black filled circles) are associated with their each individual wakelets, as predicted by linear swing amplification theory, only at distances beyond 4 disk scale-lengths where the selfgravity of the disk is lower and the individual perturbers are well-separated. As described below, we find that between two and four disk scale-lengths the arms are reinforced owing to non-linear effects and the wakelets become sufficiently overdense that they decouple from the original perturbers and then act as perturbers themselves.

To demonstrate this unexpected outcome and its radical consequences, we remove the original perturbers from the disk by replacing the giant molecular clouds with an equal mass in stars, and continue to evolve the disk, as illustrated in Fig. 9. The patterns do not disappear, contrary to the expectations of linear theory, and are instead long-lived. Because of the self-gravity of the disk, the wakes imprinted by the original perturbers become mildly self-gravitating and are of sufficiently high density to serve as perturbers themselves, thereby making the spiral features self-perpetuating. We have verified that the ensuing spiral patterns survive for at least another eight galactic years.

We emphasize that in our simulations, the spirals are selfperpetuating in a manner completely distinct from the classical density wave theory where the patterns are static or slowly rising in amplitude with time and are rigidly rotating (Lin \& Shu 1964; Bertin \& Lin 1996). Indeed, in our simulations the patterns are not global but they appear as local segments connecting together and fluctuating in amplitude with time. The evolution of the resulting spiral structure is characterized by a balance between shear and self-gravity. Locally, shear tends to stretch the waves, breaking the arms, whereas in regions where the self-gravity dominates, the pattern is overdense and generates the segments making up the arms. This local balance between shear and self-gravity gives the visual impression that the spiral structures are global patterns, but in reality they are only segments, produced by local under-dense and over-dense regions.

An alternative explanation for our results involves the possibility that such dynamical systems support actual coherent unstable modes, and that the features observed after the perturbers are removed are manifestations of these unstable modes. Thus the rapidly varying spirals seen in our simulations would result from the superposition of a few long-lived waves (for a detailed study, see Sellwood (2012)).

We generated spectrograms to test this interpretation, which supported at some level this possibility. However, our experiments were not run for a sufficiently long time to be decisive on this point. We will explore this alternative explanation with tests to discriminate between the two interpretations in a 

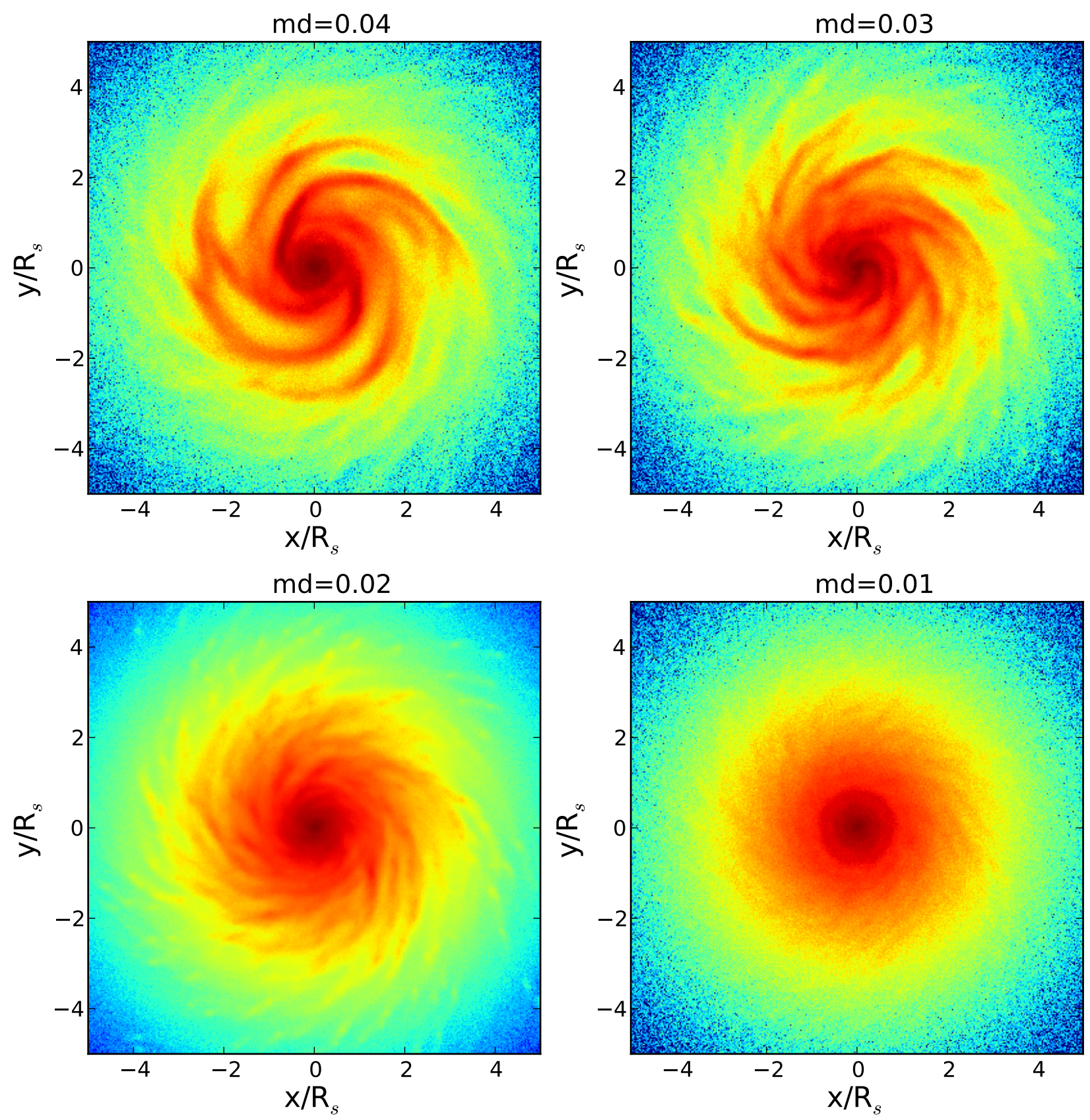

FIG. 8.- Sequence of models where we varied the contribution of the disk to the total mass distribution in the rotation curve of the galaxy. We fix the total mass of the galaxy and the mass distribution of the dark halo, but increase the disk mass fraction $m_{d}$ from a value of 0.01 to the following values: $0.02,0.03$ and 0.04. Increasing the disk fraction decreases the number of arms. Models are displayed after one galactic year.

forthcoming paper.

Our results naturally predict that the pattern speed of spiral arms in late-type spiral galaxies should not be constant but should vary with distance from the center of the galaxy. This has already been claimed for patterns speeds estimated for NGC 5164 and NGC 628 (Foyle et al. 2010; Meidt et al. 2009) and M101 (Foyle et al.2011). However, whereas these works conclude that this supports the interpretation that spiral arms are short-lived, our simulations show that the features can be long-lived as various local segments break and then later reconnect with other pieces of spiral arms, resulting in long-lived structures.

Note that we have focused on the idealized case of a rather low disk mass. These systems are stable over long periods of time and do not develop bars or arms if unperturbed. If we consider disks that are more strongly self-gravitating, by, for example, increasing the ratio of the mass of the disk to the total mass of the galaxy, $m_{d}=0.04$, the simulations favor the formation of four spiral arms as displayed at the top left panel of Fig. 8. In that event, $\lambda_{\text {crit }}$ is larger, so that longer wavelength disturbances are most strongly amplified. By examining the rotation curves of our galaxy models, we also find that those with more massive disks have lower shear rates where the bulk of their mass is located, so individual wakes can remain connected over longer distances without breaking apart owing to shear, yielding patterns with fewer global arms. Indeed the number of arms does not depend on the number of perturbers but on the structural properties of the galaxies in a 

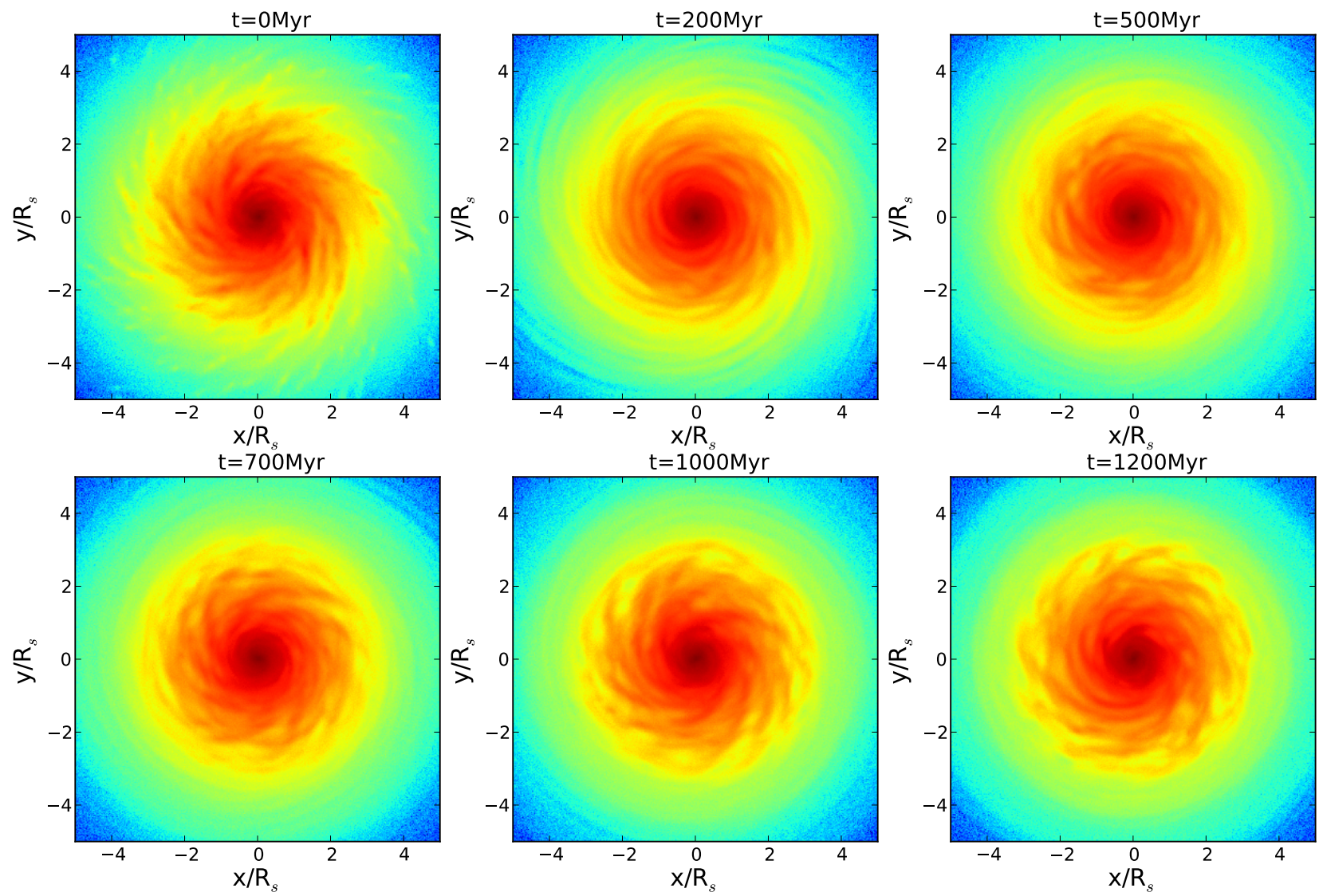

FIG. 9.- Time sequence for the evolution of spiral arms once the giant molecular clouds are removed from the disk. The left upper panel shows the stellar disk the time at which the perturbers, e.g. giant molecular clouds, are removed. We define this initial time as set to $\mathrm{t}=0$. The subsequent time evolution of the spiral arms is shown in the other panels according to the initial time definition. Each panel displays a region $30 \mathrm{kpc}$ on a side at the times indicated.

way that by changing the rotation curve of the galaxy also the number of arms varies accordingly.

In a further set of experiments, we ran simulations of disks after we systematically reduced the time at which the 1,000 perturbers, e.g. giant molecular clouds, were removed and estimated the minimum time the perturbers need to corotate with the disk to trigger the formation of spiral arms that persists even after being removed. We find that if we remove the 1,000 perturbers after $15 \mathrm{Myrs}$, this time is sufficient to trigger the process that leads later to the formation of long-lived, self-perpetuating patterns, similar to the estimates we made earlier for the required lifetimes of the original perturbers.

\subsection{Non-linear effects}

To demonstrate explicitly the possible importance of nonlinear dynamics in spiral structure formation, the top panel of Fig. 10 shows the case of a galactic disk influenced by a single perturber with a mass of $10^{7} \mathrm{M}_{\odot}$ kept cycling at 2 disk scale length, corotating with the disk, and then being removed after one galactic year. ${ }^{5}$ The image displays the disk when the perturber is still corotating with it. Initially, a wakelet is generated around the perturber in a manner consistent with the linear theory. However, non-linear effects are evident in the gravitational response of the disk to the perturbation and result in the development of additional underdense and overdense regions that are clearly visible especially in the polar coordinate plot (right top panel of Fig. 10). These features,

\footnotetext{
${ }^{5}$ The perturber is forced to cycle on a circular orbit so that it is not affected by dynamical friction.
}

which act as perturbers, are responsible for keeping alive the spiral activity and are not expected based on the linear analysis. These findings indicate that our simulations are probing an entirely new regime in which the linear theory is no longer fully applicable.

Once the non-linear features begin to develop, we remove the perturber as shown at the bottom panel of Fig. 10 and find that the wakelets do not disappear, as predicted by the linear theory. Indeed, the overdense and underdense regions act themselves as new perturbers, exciting a further response in the disk, leading to a self-perpetuating pattern in which additional unexpected features such as holes and wrapping arms in the outer regions are produced within four galactic years, as shown in Fig. 10 The expectations of linear theory are that the disk should relax after the perturbation is removed and that the spirals should gradually disappear, but we find instead that the patterns survive for many rotation periods.

We note here that the response in the disks we simulate originates at the co-rotation resonance between the perturbers and the surrounding stars. Thus, while we do not argue against the possibility that dynamical effects such as "groove instabilities" (Sellwood \& Lin 1989) can play an important role in producing spiral structure in actual galaxies, in our simulations, at least, the dominant dynamical effects seem to be associated with the response at the co-rotation resonance in the disk.

Next, we performed the same experiment with a perturber mass reduced by a factor of ten, e.g. typical of a giant molecular cloud or a globular star cluster of mass $10^{6} \mathrm{M}_{\odot}$ kept cycling at two scale lengths of the disk and checked how long 

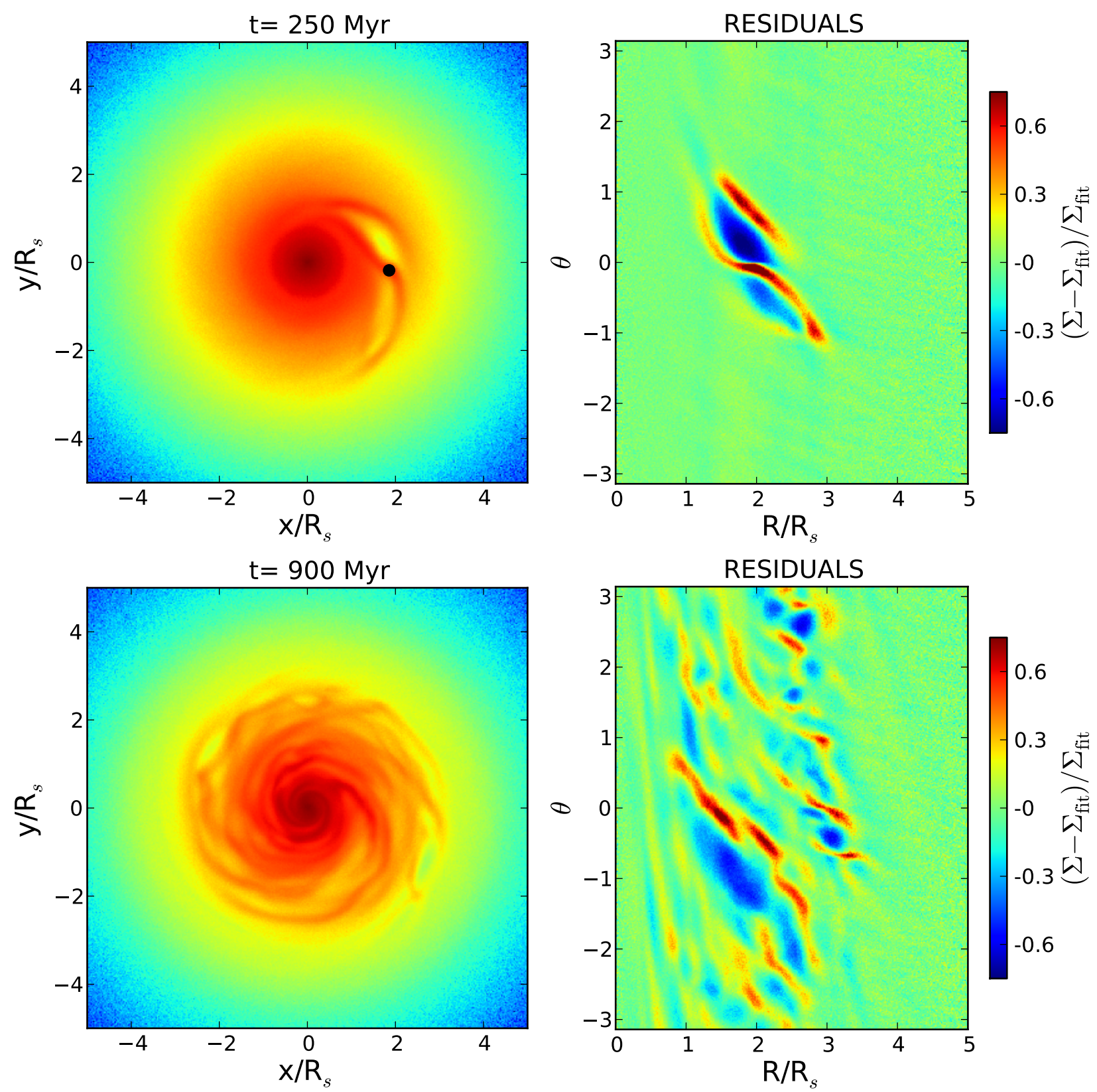

FIG. 10.-Top Panels: A stellar disk responding to a single perturber with mass of $10^{7} \mathrm{M}_{\odot}$, which is removed after one galactic year. Bottom Panels: Surface density is shown 4 galactic years after the perturber is removed confirming that the patterns survive for several rotation periods. The disk is displayed face-on in Cartesian coordinates (left panels) and surface density residuals are shown in polar coordinates (right panels).

it takes for non-linear effects to develop. The outcome is illustrated in Fig. 11 which shows that the linear approximation breaks down after roughly 3 galactic years and holes and winding arms appear following an additional 2 galactic years once the perturber is removed. This suggests that while reducing the mass of the perturber lengthens the time for non-linear effects to become important, they nevertheless still develop. Note that the amplitude of the arms (which are the overdense regions) and the depth of the holes (the underdense regions) are also reduced because they are sensitively dependent on the mass of the perturber.

\section{PREDICTIONS}

It seems clear from our results that a modest overdensity corotating with a galactic disk for a small fraction of a galactic year can trigger the formation of spiral structure that will take over and become self-perpetuating for at least several billion years. Intriguingly, observations of nearby disk galaxies suggest that when counter-rotating stellar or gas disks are present in galaxies there is no evidence of spiral structure (Bertola et al. 1999; Coccato et al. 2011), which is as expected, based on our simulations.

Furthermore, it has been argued that spiral structures are transient features that need to be re-generated by continuously adding new perturbers in the form of giant molecular clouds or clumpy gas that maintains ragged arms (Sellwood \& Carlberg 1984). Unlike simulation results of decades ago, which were compromised by limited numerical resolution, our experiments show that disks do not necessarily thicken because of the presence of spiral arms and giant molecular clouds, and that the spiral structure can persist for timescales similar to the ages of actual galactic disks. Moreover, although 

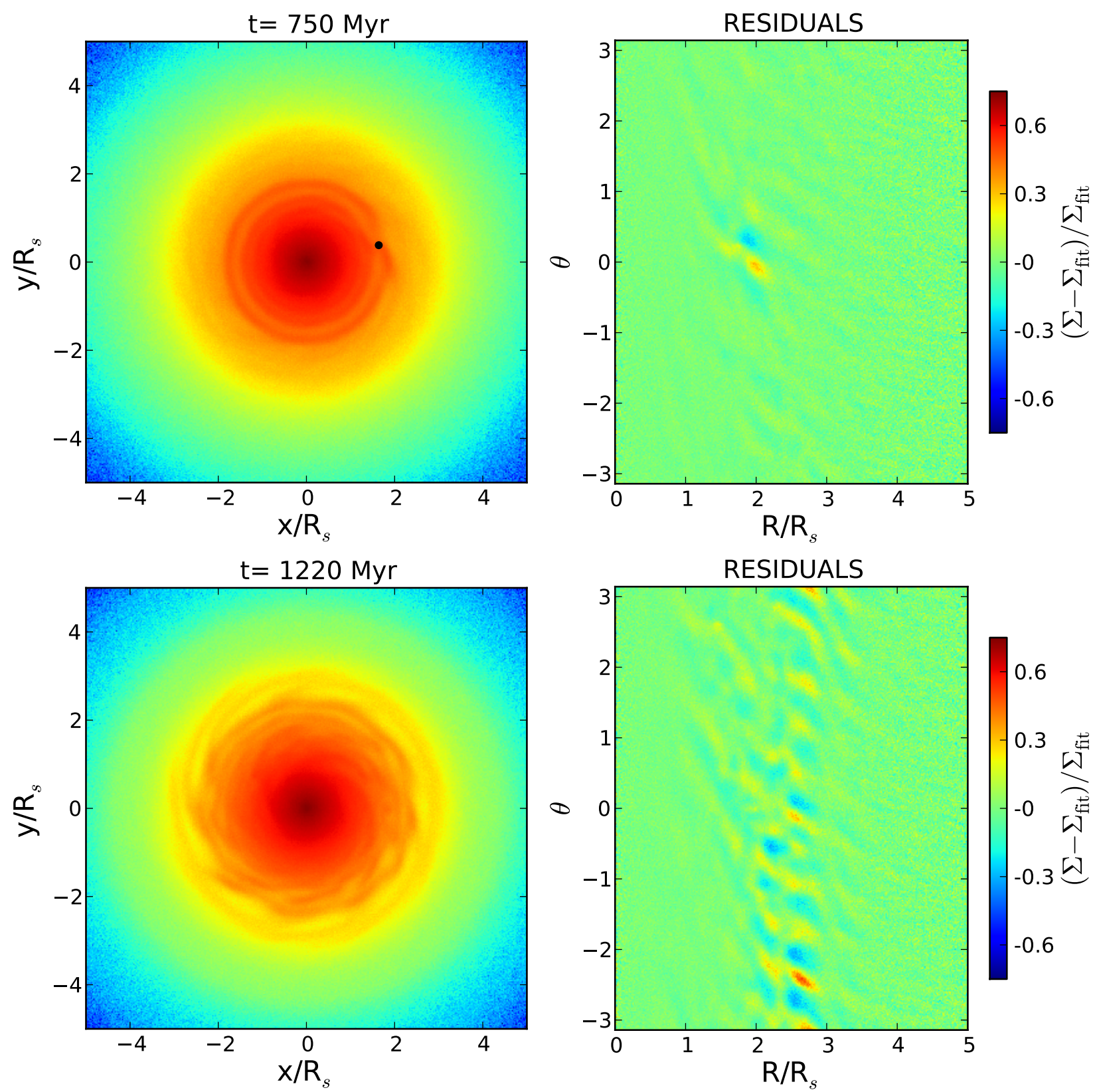

FIG. 11.- Top Panels: A stellar disk responding to a single perturber with mass of $10^{6} \mathrm{M}_{\odot}$, which is forced to remain on a circular orbit (represented by the black circle). The image displays the disk after three orbital periods computed at a distance of two scale lengths of the disk where the first non-linear effects are visible. Bottom Panels: The patterns survive for several rotation periods and surface density is shown after 2 additional galactic years once the perturber is removed. The disk is displayed face-on in Cartesian coordinates (left panels) and surface density residuals are shown in polar coordinates (right panels).

new gas accreted can excite young arms, there should be old stellar arms present in galaxies and fresh gas is not required to maintain spiral patterns over long timescales. This means that, in principle, early-type galaxies which are now gas-poor may still exhibit weak spiral features. IC 3328, an early-type dwarf galaxy with spiral structure (Lisker \& Fuchs 2009) or NGC1533 (DeGraaff et al. 2007) may be such cases.

Ultimately, other predictions of our models include definite quantitative statements regarding the pattern speeds and time-variability of actual spiral features in galaxies (Nelson, D’Onghia \& Hernquist 2012, in prep.), and the kinematic and spatial response of the stars to these fluctuating features. Spiral waves in galaxy disks churn the stars and gas in a manner that largely preserves the overall angular momentum distribution and leads to little increase in random motion. However, recent works (Sellwood \& Binney 2002) claim that changes in the angular momenta of individual stars are typically concentrated around the co-rotation radius for an individual spiral wave, but given the supposed transient nature of the waves with a wide range of pattern speeds developing in rapid succession, the entire disk should be affected. Our models indicate that spiral structures are not transient features, but eventually locally break and subsequently reconnect as a balance between the shear that stirs the arm and the self-gravity that tends to reconnect the pattern. This calls for a better understanding for how stars like the Sun can migrate if the nature and the evolution of the waves is different (Roškar et al. 2008, Minchev et al.2011)

We anticipate that there should be a correlation between the amplitude of the arms and the $Q$ parameter that defines how 
dynamically hot the disk is, in the sense that "colder" disks should, on average, display more prominent spirals. This follows from the fact that swing amplification, which is still central to our interpretation, is most vigorous in dynamically cool disks (Toomre 1981). Ongoing and planned observations (Elmegreen et al. 2011) will enable tests of our modeling in the near future, which should shed further light on the stillmysterious nature of spiral structure.

\section{CONCLUSIONS}

In this paper, we have examined the possibility that spiral structure in disk galaxies originates through the dynamical response of self-gravitating, shearing disks to local density perturbations. While our analysis has much in common with earlier studies of spiral structure formation, it differs in the following respects.

- Our N-body simulations show that spiral patterns can be described by density waves but the response of the disk to local perturbations can be highly non-linear and time-variable. Our work indicates limitations of two traditional theories that tried to explain arm formation: the theory of arms as static density waves (Lin \& Shu 1964) and linear forced-wake theory (Julian \& Toomre 1966) which were based on simplifying assumptions. In particular, our results are inconsistent with the idea that spirals are static density waves because the arms in our simulations fluctuate in time. Moreover, our results are different from what is expected based on forcedwake theory because we show that the arms can be long-lived and are not necessarily transients even when the original perturbations responsible for driving the patterns are removed.

- The spiral patterns in our simulations are not global as predicted by the classical static density wave theory but locally they appear to fluctuate in amplitude with time. It is the local balance between the shear that stirs waves, breaking the arms, and the self-gravity that makes up the arms that gives the visual impression that the patterns are global whereas they are actually segments produced by local under-dense and over-dense regions, at least for relatively low mass disks. These under-dense and over-dense regions act as perturbers, keep alive the spiral activity and probe an entirely new regime in which the linear theory is no longer fully applicable.

- The dynamical response in the disk originates at the corotation resonance between the perturbers and the surrounding stars. This implies that our features that wrap up and get replaced statistically have angular speeds that are not constant with location, but decrease with radius. The feature speed slightly differs from the surrounding stars, explaining why the arms do not wind up while being at the same time long-lived.

- A modest overdensity corotating for at least 25 million years will excite a response in the disk that will lead to a self-perpetuating pattern owing to non-linear effects.

We believe that there is already some observational support for our picture. In particular, our model predicts that there should exist red disk galaxies with no gas but that may still have arms. Galaxies such as this have been discovered (Masters et al. 2010) and current theories for spiral arm formation do not explain their morphologies. In our model, the pattern speeds of the spiral features should vary with location within a disk, as has been claimed for some nearby spirals (Foyle et al. 2010, 2011, Meidt et al. 2009). We also predict that the number of arms in galaxies should depend on the structural properties of the galaxy and that there will be a correlation between the amplitude of the arms and how dynamically cold the disk is, in the sense that colder disks should display more prominent arms. These predictions are readily testable and relevant observational studies should provide strong tests of our model in the forthcoming years.

\section{APPENDIX}

\section{STATIC POTENTIAL FOR A THIN EXPONENTIAL DISK}

To verify the importance of self-gravity in the formation of arms, we performed an N-body simulation of a disk of stars treated as test particles. In order to treat the stars as test particles, we compute the forces acting on them by introducing a static potential for a thin exponential disk of the form:

$$
\begin{aligned}
& a_{x}=-\cos \theta \frac{\partial \Phi}{\partial R} \\
& a_{y}=-\sin \theta \frac{\partial \Phi}{\partial R} \\
& a_{z}=-\frac{\mathrm{GM}_{*}}{R_{h}^{2}} \exp \left(-R / R_{h}\right) \tanh \left(\frac{z}{z_{0}}\right)
\end{aligned}
$$

where $\theta$ is the angle phase and

$$
\begin{aligned}
\frac{\partial \Phi}{\partial r}= & -\frac{G M_{*} z_{0}}{R_{h}^{2}} \exp \left(-R / R_{h}\right)\left(\frac{1}{R_{h}}\right) \ln \cosh \left(\frac{\mathrm{z}}{\mathrm{z}_{0}}\right) \\
& -\frac{G M_{*}}{2 R_{h}}\left[I_{0}(y) K_{1}(y)-I_{1}(y) K_{0}(y)\right] \\
& -\frac{G M_{*} y}{2 R_{h}^{2}}\left[I_{0}^{\prime}(y) K_{1}(y)+I_{0}(y) K_{1}^{\prime}(y)+\right. \\
- & \left.I_{1}^{\prime}(y) K_{0}(y)-I_{1}(y) K_{0}^{\prime}(y)\right]
\end{aligned}
$$

The interactions between the perturbers and stars are computed directly, meaning that the stellar orbits are influenced by these gravitational interactions, as in the simulations with disk self-gravity presented above. 


\section{AMPLIFICATION PARAMETER FOR A GENERAL DISK GALAXY}

To interpret the results shown in $\S 4.4$ we consider the Toomre $X$-parameter for a galaxy like that in our simulations, using

$$
X=\frac{\kappa^{2}}{2 \pi G \Sigma} \frac{R}{m} .
$$

In general, the epicyclic frequency depends on the total angular frequency which is the sum of the angular frequencies of all the components of a galaxy, in particular the bulge, disk and dark halo, according to

$$
\Omega^{2}=\Omega_{D}^{2}+\Omega_{B}^{2}+\Omega_{H}^{2} .
$$

For an exponential disk:

$$
\Omega_{D}^{2}=\frac{G M_{D}}{2 R_{h}^{3}}\left[I_{0}(y) K_{0}(y)-I_{1}(y) K_{1}(y)\right]
$$

where $y=R / 2 R_{h}, R_{h}$ is the disk scale length and $M_{D}$ the disk total mass. For bulge and halos described by Hernquist models, the angular frequency of the bulge is

$$
\Omega_{B}^{2}=\frac{G M_{B}}{\left(R+a_{b}\right)^{2}},
$$

where $a_{b}$ and $M_{B}$ are the bulge scale length and mass, respectively and for the halo

$$
\Omega_{H}^{2}=\frac{G M_{H}}{\left(R+a_{h}\right)^{2}}
$$

where $a_{h}$ and $M_{H}$ are the halo scale length and the mass, respectively. The amplification parameter for a general disk galaxy becomes:

$$
\begin{aligned}
X= & \frac{e^{2 y}}{m}\left(\left[\frac{M_{B}}{M_{D}} \frac{2 y+3 a_{b} / R_{h}}{\left(2 y+a_{b} / R_{h}\right)^{3}}\right]\right. \\
& +\left[\frac{M_{H}}{M_{D}} \frac{2 y+3 a_{h} / R_{h}}{\left(2 y+a_{h} / R_{h}\right)^{3}}\right] \\
& +\frac{y^{2}}{2}\left(3 I_{1} K_{0}-3 I_{0} K_{1}+I_{1} K_{2}-I_{2} K_{1}\right) \\
& \left.+4 y\left(I_{0} K_{0}-I_{1} K_{1}\right)\right)
\end{aligned}
$$

where $m$ characterizes the Fourier coefficient $A_{m}$ and indicates the number of arms. Note that substituting the values for the structural parameters of the galaxy model adopted here into eq. (B6) gives an estimate of the number of arms as $\sim 7$, in accord with our simulations.

We are grateful to the referee Jerry Sellwood for constructive suggestions and to Alar Toomre for much generous and wise advice. We thank Mark Reid and Debora Sijacki for a careful reading of the manuscript. Numerical simulations were performed on the Odyssey supercomputer at Harvard University.

\section{REFERENCES}

Athanassoula, E. 1984, Phys. Rep., 114, 319

Bertin, G., \& Lin, C. C. 1996, Spiral structure in galaxies a density wave theory, ed. Bertin, G. \& Lin, C. C.

Bertola, F., Corsini, E. M., Vega Beltrán, J. C., Pizzella, A., Sarzi, M., Cappellari, M., \& Funes, J. G. 1999, ApJ, 519, L127

Binney, J., \& Lacey, C. 1988, MNRAS, 230, 597

Binney, J., \& Tremaine, S. 2008, Galactic Dynamics: Second Edition, ed. Binney, J. \& Tremaine, S. (Princeton University Press)

Carlberg, R. G., \& Freedman, W. L. 1985, ApJ, 298, 486

Coccato, L., Morelli, L., Corsini, E. M., Buson, L., Pizzella, A., Vergani, D., \& Bertola, F. 2011, MNRAS, 412, L113

DeGraaff, R. B., Blakeslee, J. P., Meurer, G. R., \& Putman, M. E. 2007, ApJ, 671,1624

D’Onghia, E., Springel, V., Hernquist, L., \& Keres, D. 2010a, ApJ, 709, 1138

D'Onghia, E., Vogelsberger, M., Faucher-Giguere, C.-A., \& Hernquist, L. 2010b, ApJ, 725, 353

Efstathiou, G., Lake, G., \& Negroponte, J. 1982, MNRAS, 199, 1069

Elmegreen, D. M., Elmegreen, B. G., Yau, A., Athanassoula, E., Bosma, A., Buta, R. J., Helou, G., Ho, L. C., Gadotti, D. A., Knapen, J. H., Laurikainen, E., Madore, B. F., Masters, K. L., Meidt, S. E., MenéndezDelmestre, K., Regan, M. W., Salo, H., Sheth, K., Zaritsky, D., Aravena, M., Skibba, R., Hinz, J. L., Laine, J., Gil de Paz, A., Muñoz-Mateos, J.-C., Seibert, M., Mizusawa, T., Kim, T., \& Erroz Ferrer, S. 2011, ApJ, 737, 32
Foyle, K., Rix, H.-W., Dobbs, C. L., Leroy, A. K., \& Walter, F. 2011, ApJ, 735, 101

Foyle, K., Rix, H.-W., Walter, F., \& Leroy, A. K. 2010, ApJ, 725, 534

Fujii, M. S., Baba, J., Saitoh, T. R., Makino, J., Kokubo, E., \& Wada, K. 2011, ApJ, 730, 109

Gauthier, J.-R., Dubinski, J., \& Widrow, L. M. 2006, ApJ, 653, 1180

Goldreich, P., \& Lynden-Bell, D. 1965, MNRAS, 130, 125

Grand, R. J. J., Kawata, D., \& Cropper, M. 2012, MNRAS, 2358

Hernquist, L. 1990, ApJ, 356, 359

-. 1993, ApJ, 409, 548

Hernquist, L., \& Katz, N. 1989, ApJS, 70, 419

Julian, W. H., \& Toomre, A. 1966, ApJ, 146, 810

Lin, C. C., \& Shu, F. H. 1964, ApJ, 140, 646

Lisker, T., \& Fuchs, B. 2009, A\&A, 501, 429

Masters, K. L., Mosleh, M., Romer, A. K., Nichol, R. C., Bamford, S. P., Schawinski, K., Lintott, C. J., Andreescu, D., Campbell, H. C., Crowcroft, B., Doyle, I., Edmondson, E. M., Murray, P., Raddick, M. J., Slosar, A., Szalay, A. S., \& Vandenberg, J. 2010, MNRAS, 405, 783

Meidt, S. E., Rand, R. J., \& Merrifield, M. R. 2009, ApJ, 702, 277

Minchev, I., Famaey, B., Combes, F., Di Matteo, P., Mouhcine, M., \& Wozniak, H. 2011, A\&A, 527, A147

Purcell, C. W., Bullock, J. S., Tollerud, E. J., Rocha, M., \& Chakrabarti, S. 2011, Nature, 477, 301 
Roškar, R., Debattista, V. P., Quinn, T. R., Stinson, G. S., \& Wadsley, J. 2008, ApJ, 684, L79

Sellwood, J. A. 2000, Ap\&SS, 272, 31

-. 2011, MNRAS, 410, 1637

-. 2012, ApJ, 751, 44

Sellwood, J. A., \& Binney, J. J. 2002, MNRAS, 336, 785

Sellwood, J. A., \& Carlberg, R. G. 1984, ApJ, 282, 61

Sellwood, J. A., \& Lin, D. N. C. 1989, MNRAS, 240, 991

Springel, V. 2000, MNRAS, 312, 859

—. 2005, MNRAS, 364, 1105
Springel, V., Di Matteo, T., \& Hernquist, L. 2005, MNRAS, 361, 776

Toomre, A. 1964, ApJ, 139, 1217

-. 1977, ARA\&A, 15, 437

Toomre, A. 1981, in Structure and Evolution of Normal Galaxies, ed. S. M. Fall \& D. Lynden-Bell, 111-136

-. 1990, Gas-hungry Sc spirals., ed. Wielen, R., 292-303

Toomre, A., \& Kalnajs, A. J. 1991, in Dynamics of Disc Galaxies, ed. B. Sundelius, 341 